[3] Lipstman, S.; George, S.; Muniappan, S.; Goldberg, I., 2007, submitted for publication

\section{MS13 O4}

Reproducibility in inorganic crystal engineering: A cautionary tale Susan A Bourne ${ }^{\text {a }}$, Lesego J Moitsheki, Gaelle Ramon, Laura C Trollip. Department of Chemistry, University of Cape Town, Rondebosch 7701, South Africa E-mail: susan.bourne@uct.ac.za

Keywords: coordination chemistry compounds, powder diffraction, thermal analysis.

One of the aims of crystal engineering is to produce crystalline materials with predictable and reproducible supramolecular interactions. While the most common of the latter is probably the hydrogen bond (often used to generate organic as well as inorganic frameworks) a number of other interactions are also possible. However, changes in experimental conditions may have a strong influence on the materials produced, making reproducibility difficult to achieve.

We have recently prepared a series of metal-organic framework and network materials using a series of phenylamine and pyridine derivatives with transition metals and lanthanides. The same component compounds can be used to prepare network structures, inorganicorganic layered structures or even simple salts. Changes in conditions (eg. $\mathrm{pH}$ or temperature) are critical in determining the type of material obtained. This paper will discuss some of the well-ordered metal organic networks and layered compounds we have prepared, with a particular emphasis on those produced using $\mathrm{Co}\left(\mathrm{NO}_{3}\right)_{2}$ and 4,4'-bipyridine-N,N'-dioxide and those produced using a novel scorpion-like ligand. We have found that moderation of reaction temperature and variation in solvent mixtures results in a wide variety of different crystalline compounds being formed. Compounds have been characterised by single crystal and powder X-ray diffraction. Their thermal stability and decomposition behaviour have been studied using Differential Scanning Calorimetry, Thermogravimetry and Hot Stage Microscopy. Guest-exchange reactions in channel compounds have been followed by means of powder x-ray diffraction.
MS13 05

Crystal Structures and Properties of Organically Templated Metal Phosphates and Metal Phosphites Sue-Lein Wang, Department of Chemistry, National Tsing Hua University. Hsinchu, Taiwan, ROC

E-mail: slwang@mx.nthu.edu.tw

Keywords: Open-framework structure, zinc phosphate, zinc phosphite

Organic-inorganic hybrid metal phosphates have exhibited a variety of interesting open-framework structures as well as a wide range of applications such the traditinal use in catalysis, gas sorption and ion exchange and modern modern low- $k$ materials, zeolite-dye micro-laser, or even inorganic phosphor. Recently, we have investigated the tetrahedral zinc phosphate materials and discovered several phases with distinctive microporous structures and interesting photo luminescence property. Herein, we report the NTHU-n series $(n=1-5)$ which include a neutral 24ring channel structure, a novel three-dimensional highly porous organic-inorganic hybrid framework with bimodal porosity, an elastic layered lattice with capability of molecular recognition by encapsulating supramolecular molecules, a unique mixed-metal zincophosphate with intrinsic yellow/white emission, and the first 26-ring channel structure of mixed metal phosphites.

[1] Liao, Y. C.; Lin, C. H.; Wang, S. L. J. Amer. Chem. Soc. 2005, 127, 9986.

[2] Liao, Y. C.; Jiang, C. J.; Wang, S. L. J. Amer. Chem. Soc. $2005,127,12794$

[3] Liao, Y. C., Liao, F.L., Chang, W.K., Wang, S.L. J. Amer. Chem. Soc, 2004, 126, 1320.

[4] Lin, C.H.; Yang, Y.C.; Chen, C.Y.,.Wang, S. L. Chem. Mater. 2006, 18, 2095-2101

[5] Lin, C. H.; Wang, S. L.; Lii, K. H. J. Amer. Chem. Soc. 2001, 123,4649 . 